\title{
High Frequency of Human Leukocyte Antigen-B*57:01 Allele Carriers among HIV-Infected Patients in Serbia
}

\author{
Marina Siljic ${ }^{a}$ Dubravka Salemovic ${ }^{b}$ Valentina Cirkovic ${ }^{a}$ Ivana Pesic-Pavlovic ${ }^{c}$ \\ Marija Todorovic ${ }^{a}$ Jovan Ranin ${ }^{a}$ b Gordana Dragovic ${ }^{a}$ Djordje Jevtovic $^{a}{ }^{b}$ \\ Maja Stanojevic ${ }^{a}$ \\ a University of Belgrade School of Medicine, ${ }^{b}$ HIV/AIDS Unit, Clinical Center Serbia, University Hospital of Infectious \\ and Tropical Diseases, and 'Department of Virology, Clinical Center Serbia, Belgrade, Serbia
}

\section{Keywords}

Antiretroviral drugs · HLA-B*57:01 · HIV infection · Abacavir · Serbia

\begin{abstract}
Abacavir is an effective antiretroviral drug and one of the most commonly used nucleoside reverse transcriptase inhibitors in Serbia. A percentage of the treated patients experience a potentially life-threatening hypersensitivity reaction, which was shown to be associated with the presence of the class I MHC allele, HLA-B*57:01; hence genotyping for HLA-B*57:01 prior to starting abacavir is nowadays recommended in international HIV treatment guidelines. In Serbia, this testing became available in 2013. This study was designed to estimate the prevalence of the HLA-B*57:01 allele in Serbian HIV-1-infected patients. The presence of the HLA-B*57:01 allele was analyzed in 273 HIV-1-infected patients aged 18 years or more, who were abacavir naïve. Buccal swab samples were obtained from all participants and assayed for the presence of HLA-B*57:01 using a commercially available HLA-B*57:01 real-time PCR kit. The presence of the HLA-B*57:01 allele was found in 22 of 273 tested individuals (8\%; $95 \% \mathrm{Cl} 5.4-11.9 \%)$. This is the first study that
\end{abstract}

\section{KARGER}

(C) 2017 S. Karger AG, Basel

E-Mail karger@karger.com

www.karger.com/int estimated the HLA-B*57:01 prevalence among HIV-infected patients in Serbia. The very high prevalence of HLA-B*57:01 found in our study strongly supports HLA-B*57:01 genotyping, which should be implemented prior to the initiation of an abacavir-containing therapy to reduce the risk of potentially life-threatening hypersensitivity reactions.

(c) 2017 S. Karger AG, Basel

\section{Significance of the Study}

Abacavir remains a key drug of antiretroviral regimens in Serbia. Although generally well tolerated, a percentage of patients experience a potentially life-threatening hypersensitivity reaction (HSR) associated with the class I MHC allele HLA-B*57:01. We present the first study estimating HLA-B*57:01 prevalence among HIV-infected patients in Serbia. Our findings strongly support HLA-B*57:01 genotyping prior to initiation of an abacavir-containing therapy.

Marina Siljic and Dubravka Salemovic contributed equally to this work.
Maja Stanojevic, $\mathrm{MD}, \mathrm{PhD}$

University of Belgrade School of Medicine

Dr Subotica 1

RS-11000 Belgrade (Serbia)

E-Mail mstanojevic@ med.bg.ac.rs 


\section{Introduction}

Abacavir is an antiretroviral drug of the nucleoside reverse transcriptase inhibitor (NRTI) class, which is commonly combined with antiretroviral therapy for treating $\mathrm{HIV}$ infection. Abacavir acts as a potent guanosine nucleoside inhibitor of reverse transcriptase [1]. It is generally well tolerated, and common side effects include nausea, headache, and diarrhea [2]. However, approximately $5-8 \%$ of patients experience HSR within the first 6 weeks of treatment, which can be severe and potentially lifethreatening. HSR includes at least two of the following symptoms: fever, rash, cough, gastrointestinal symptoms (e.g., nausea, vomiting, or abdominal pain), dyspnea, and fatigue $[3,4]$. HLA-B*57:01, a specific human leukocyte antigen allele, has been shown to represent the strongest predictor of ABC-HSR $[5,6]$. Recently, the mechanism of this association has been explained as specific binding of the abacavir molecule to the HLA-B*57:01 protein at its "F-pocket." The resulting protein complex thus becomes recognized as a foreign body, triggering an autoimmune activation by CD8+ T cells, with ABC-HSR as the outcome $[7,8]$.

Screening for HLA-B*57:01 prior to initiation of abacavir therapy is currently recommended by leading international guidelines, and patients testing positive for the HLA-B*57:01 allele should not be prescribed abacavir [9]. Regarding the most frequent HLA alleles, there is widespread variation according to race and ethnicity in the prevalence of the HLA-B*57:01 allele among human populations. The highest prevalence is in Caucasian populations (5-8\%), whereas in African-American, Asian, and Hispanic populations, the prevalence is significantly lower $(0.26-3.6 \%)$ [10, 11$]$.

The present study aimed to assess the prevalence of the HLA-B*57:01 allele among HIV-infected patients in Serbia.

\section{Patients and Methods}

\section{Study Population}

The study included 273 consenting, HIV-infected patients aged 18 years or more, referred to the HIV/AIDS Unit of the University Hospital for Infectious and Tropical Diseases in Belgrade, which is the main treatment center for most HIV-infected patients in Serbia. Epidemiological, clinical, and behavioral data were collected using a standardized questionnaire. Written consent for participation was obtained from all participants, and the study was approved by the local ethics committee.
Table 1. General clinical and demographic data of the study population

$\begin{array}{lc}\text { Gender, } n & \\ \text { Male } & 240(87.9 \%) \\ \text { Female } & 33(12.1 \%) \\ \text { Transmission route, } n & \\ \text { MSM } & 148(54.2 \%) \\ \text { Heterosexual } & 59(21.6 \%) \\ \text { Intravenous drug use } & 18(6.6 \%) \\ \text { Vertical transmission } & 2(0.6 \%) \\ \text { Unknown } & 46(16.9 \%) \\ \text { Place of residence, } n & \\ \text { Urban area } & 171(62.6 \%) \\ \text { Rural } & 56(20.5 \%) \\ \text { Unknown } & 46(16.9 \%) \\ \text { CDC disease stage, } n & \\ \text { A } & 140(51.2 \%) \\ \text { B } & 45(16.5 \%) \\ \text { C } & 42(15.4 \%) \\ \text { Unknown } & 46(16.9 \%) \\ \text { OSTI, } n & \\ \text { Positive } & 38(13.9 \%) \\ \text { Negative } & 189(69.2 \%) \\ \text { Unknown } & 46(16.9 \%) \\ \text { Median age, years } & \\ \text { Study patients } & 39.27 \pm 13.2 \\ \text { Male } & 38.67 \pm 13 \\ \text { Female } & 39.5 \pm 12.3 \\ \text { Positive for HLA-B*5701 } & 22 / 273(8.0 \%) \\ \text { 95\% CI } & 5.4-11.9 \%\end{array}$

MSM, men who have sex with men; OSTI, presence/history of other sexually transmitted illness.

Sample Collection and Preparation

From January 2014 to April 2015, buccal swab samples were collected from patients who were abacavir naïve, with or without previous exposure to therapy regimes with other antiretrovirals. Two buccal swabs (in $1 \mathrm{~mL}$ of PBS each) were obtained per patient for this analysis. The samples were centrifuged for $10 \mathrm{~min}$ at 1,000 rpm at room temperature.

DNA Extraction and Real-Time PCR Analysis

After carefully removing $800 \mu \mathrm{L}$ of the supernatant, the pellet was resuspended in a remaining $200-\mu \mathrm{L}$ volume of the supernatant and immediately used for DNA extraction, or stored at $-80^{\circ} \mathrm{C}$ until further processing.

DNA was extracted using the Pure Link Genomic DNA Mini Kit (Invitrogen, Waltham, MA, USA) according to the manufacturer's instructions. In brief, proteinase $\mathrm{K}$, and Pure Link Genomic Lysis Buffer were added to the pellet and incubated at $55^{\circ} \mathrm{C}$ for 10 min. Subsequently, 96-100\% ethanol was added, and the whole content carefully transferred onto Pure Link Spin Column. The columns were centrifuged at 10,000 and 14,000 rpm for 1 and $3 \mathrm{~min}$, respectively, with serial addition of wash buffers 1 and 2
44

Intervirology 2017;60:43-47

DOI: $10.1159 / 000477810$
Siljic et al. 
and final elution using elution buffer. The obtained eluate was frozen at $-80^{\circ} \mathrm{C}$ and used for real-time PCR.

Real-time PCR analysis was performed with the HLA-B*57:01 Real-TM PCR kit according to the manufacturer's protocol (Sacace Biotecnologies Srl, Como, Italy). The HLA-B*57:01 Real-TM PCR kit is a qualitative TaqMan-based real-time amplification kit with HLA-B*57:01 allele-specific primers, containing also primers for the human $\beta$-globin gene as the internal control.

The sample size was calculated based on the assumed HLAB*57:01 phenotype prevalence of about $6 \%$ in the Serbian population; therefore, a sample of not less than 200 subjects was planned for enrollment, ensuring a maximum width of the observed $95 \%$ confidence interval $\leq 3.5 \%$. Obtained results were processed using standard statistical analysis.

\section{Results}

The study included 273 HIV-infected patients who fulfilled the inclusion criteria. All patients were abacavir naïve. The patients presented in different stages of HIV disease: of the 273 patients, 140 patients were in CDC stage A (51.3\%), 45 in stage B (16.5\%), whereas 42 patients were in CDC stage C (15.4\%). General clinical and demographic characteristics of the studied patients are presented in Table 1. Patients were mostly male (87.9\%), while the most common transmission risk was men who have sex with men (54.2\%).

The presence of the HLA-B*57:01 allele was found in 22 of the 273 tested individuals (8\%, 95\% CI 5.4-11.9\%).

\section{Discussion}

Here, we present the first comprehensive study of the prevalence of the HLA-B*57:01 allele among HIV-infected patients in Serbia.

Serbia remains a country with a low prevalence of HIV infection $(<0.1 \%)$, although in Serbia HIV is known to be present for more than 3 decades, as the first cases were reported among intravenous drug users in 1985 [12]. According to the latest epidemiological data, the cumulative number of reported cases exceeded 3,000, with an incidence rate of HIV-1 infection of around 15 per million in 2014 [13]. The proportion of intravenous drug users among newly diagnosed HIV infections has significantly decreased, while the infection rate among men who have sex with men has risen sharply, becoming the most prevalent transmission route among newly diagnosed infections in Serbia, with around 60\% in 2014. General characteristics of the study population (sex, place of origin, disease stage, and route of infection)

HLA-B*57:01 Allele in HIV-Infected Serbians corresponded to the data in the Serbian National Database (http://www.batut.org.rs/index.php? content = 1095), which indicated the representative nature of the collected sample.

Antiretroviral therapy has been available in Serbia since 1987, starting with zidovudine monotherapy; HAART was introduced in 1997. However, the available repertoire of antiretroviral drugs fully covered by the national health insurance system remains rather limited [14]. Currently, therapeutic options available in Serbia for initial HIV treatment include zidovudine, lamivudine, and abacavir among the NRTIs; nevirapine and efavirenz among the non-NRTIs; and saquinavir, fosamprenavir, and lopinavir/ritonavir among the protease inhibitors. Some of the newer antiretroviral drugs, namely maraviroc, raltegravir, darunavir, and even tenofovir, although licensed worldwide since 2004, have only recently, in 2014, become available in Serbia; however, they are still restricted to patients with proven resistance to previous antiretroviral therapy. Hence, abacavir is among the most commonly used NRTIs in Serbia as first-line and subsequent regimens [15]. In Serbia, testing for the presence of the HLA-B*57:01 allele became available in 2013.

Prior to the initiation of abacavir-containing therapy, testing for the presence of the HLA-B*57:01 allele is recommended by leading international guidelines to identify patients who are at risk of developing HSR [9]. Large studies established the effectiveness of prospective HLAB*57:01 screening to prevent HSR to abacavir, with a negative predictive value of $100 \%$, whereas the positive predictive value was found to be much lower $[16,17]$. This is partly due to the mechanism underlying abacavirinduced HSR, which is explained by steric binding of the drug to the HLA-B*57:01 protein by specific amino-acid residues in its "F-pocket," resulting in the protein complexing with self-peptides, of which some become recognized as foreign $[7,8]$. A similar mechanism is speculated to be implicated in other drug-induced HLA-associated hypersensitivity $[18,19]$. However, abacavir HLA-B*57:01 penetrance was not absolute and possibly linked to differing spectrums of self-tolerant peptides in the different populations. Hence, the positive predictive value of HLA-B*57:01 genotyping is estimated to be around $50 \%$, which means that a proportion of patients that is denied abacavir on the basis of their genotyping results would actually benefit from abacavir without experiencing an HSR $[3,4]$. This also leads to significant variation in the HLA-B*57:01 allele frequency depending on the HIV patients' race and ethnicity; generally,

Intervirology 2017;60:43-47 DOI: $10.1159 / 000477810$ 
white Caucasians have an incidence of around 6\% versus only about $2.5 \%$ in the black population [20]. Reported country-specific data in Europe also vary widely, from rather low (Portugal 1.3\% and Finland 2\%), moderate (UK 3.75\%, Ireland 4.82\%, Spain 5.97\%, France 5.33\%, and the Netherlands 6.12\%) to rather high (Germany 7.19\% and Switzerland 8.79\%) [18]. The overall estimate of the HLA-B*57:01 prevalence in Europe was found to be $4.98 \%$ [11]. In Europe, an increase in HLA-B*57:01 phenotype frequency, from east to west, has been described, from a prevalence of $1.4 \%$ in Romania and $4.04 \%$ in the European part of Russia to a prevalence of $6.9 \%$ in southern France, $7.5 \%$ in Northern Ireland, and 11.2\% in the Republic of Ireland [21, 22]. In particular, literature data about the prevalence of HLA-B*57:01 carriers in countries of Central and Southeast Europe are rather scarce, e.g., in Croatia, Poland, and the Czech Republic, where prevalence estimates of $4.7,4.7$, and $5.08 \%$, respectively, have been reported [23-25]. Existing literature data about the HLA-B*57:01 prevalence in Serbia include those reported in the allele frequency database, i.e., $6.9 \%$, and preliminary data related to HIV RT 245 codon polymorphisms $(6.5 \%)[21,26]$. The prevalence of the HLA-B*57:01 allele of $8 \%$ found in our study ranks among the highest ones reported in Europe [11]. Otherwise, a recent study analyzing 5 HLA locus polymorphisms and haplotype frequencies in the Serbian population characterized the Serbian population as closely related to populations living in central Balkan and neighboring European regions [27]. In spite of the high prevalence of HLA-B*57:01 in HIV-infected patients in Serbia and the widespread use of abacavir among them, abacavir-related HSR has only rarely been clinically recorded in Serbia. This discrepancy implies a role of additional genetic risk factors in abacavir-related HSR. Apart from abacavir HSR, the presence of HLA-B*57:01 in HIV-infected patients has been implicated in slower disease progression, although the risk of progression may vary among patients carrying this allele, as also shown for HLA alleles in other viral infections, such as hepatitis C [28]. Further, a recent study of HLA-related mate choice implied its behavioral relevance in humans, with HLA similarity affecting different aspects of partnership [29].

The HLA locus is inherently polymorphic, with many highly similar alleles, and subtle differences make their discrimination from one another very difficult. Nowadays, a number of high-resolution molecular techniques are being used for the detection of the HLA-B*57:01 allele, including sequence-specific oligonucleotide probe hybridization, DNA sequence-based typing, sequencespecific primer PCR, flow cytometry, allele-specific PCR, and real-time PCR [19]. In our study, we used commercially available real-time PCR, which is considered a fast and reliable method to accurately amplify and analyze genomic DNA [19]. Knowing the heterogeneity of HLA alleles, i.e., the latest release (3.21.0) of the IMGT/HLA database (https://www.ebi.ac.uk/cgi-bin/ ipd/imgt/hla/allele.cgi) of July 2015 lists 22 sequences deposited for HLA-B*57:01 (B*57:01:01-B*57:01:22), constant vigilance in updating and improving detection methods is needed. One could speculate that the rather high prevalence of HLA-B*57:01 in HIV-infected patients in Serbia found in our study might be attributed to false-positive detection, as described in some previous studies [11]. However, existing studies comparing commercially available real-time PCR tests for HLA$B * 57: 01$ detection demonstrated their high concordance and reliability, and there is only a single report of possibly false-negative results published to date [30]. On the other hand, the use of buccal swabs as primary samples facilitated easy access of a noninfective source of DNA in our study.

Of note, corresponding to the data in the Serbian National Database of 3.6:1, the male/female ratio in our study group allowed for relevant conclusions regarding the HIV-infected population (http://www.batut.org. rs/index.php? content $=1095)$. However, this ratio differed from the one in the general population of Serbia (1:1.05), and hence direct extrapolation to the Serbian general population was not possible [31].

Pretreatment screening for HLA-B*57:01 prior to abacavir-containing therapy, to prevent abacavir-induced HSR, has been repeatedly reported to be cost-effective $[32,33]$. Recently, the cost-effectiveness of HLA-B*57:01 genotyping related to prescribing abacavir has been questioned, in particular in regions and populations with low prevalence $[34,35]$. The cost-effectiveness of pharmacogenomic screening is influenced by many factors, with allele frequency and availability of laboratory tests being some of them.

\section{Conclusion}

In view of the evident benefits of $B^{*} 57: 01$ screening prior to abacavir therapy initiation, its high prevalence in the population of HIV-infected patients in Serbia, and the availability of a proper screening method, $B^{*} 57: 01$ typing in HIV-infected patients in Serbia is strongly recommended.
46

Intervirology 2017;60:43-47 DOI: $10.1159 / 000477810$
Siljic et al. 


\section{Acknowledgment}

This work was partially funded by the Ministry of Education and Science of the Republic of Serbia (grant No. 175024) and grant support for acquisition of reagents for HLA testing by GSK.

\section{Disclosure Statement}

The authors declare to have no conflicts of interest.

\section{References}

1 Melroy J, Nair V: The antiviral activity, mechanism of action, clinical significance and resistance of abacavir in the treatment of pediatric AIDS. Curr Pharm Des 2005;11:38473852.

2 Yuen GJ, Weller S, Pakes GE: A review of the pharmacokinetics of abacavir. Clin Pharmacokinet 2008;47:351-371.

3 Martin MA, Klein TE, Dong BJ, Pirmohamed M, Haas DW, Kroetz DL: Clinical pharmacogenetics implementation consortium guidelines for $H L A-B$ genotype and abacavir dosing. Clin Pharmacol Ther 2012;91:734-738.

4 Martin MA, Hoffman JM, Freimuth RR, et al: Clinical pharmacogenetics implementation consortium guidelines for HLA-B genotype and abacavir dosing: 2014 update. Clin Pharmacol Ther 2014;95:499-500.

5 Hetherington S, Hughes AR, Mosteller M, et al: Genetic variations in HLA-B region and hypersensitivity reactions to abacavir. Lancet 2002;359:1121-1122.

6 Mallal S, Phillips E, Carosi G, et al: HLA$B^{*} 5701$ screening for hypersensitivity to abacavir. N Engl J Med 2008;358:568-579.

7 Illing PT, Vivian JP, Dudek NL, et al: Immune self-reactivity triggered by drug-modified HLA-peptide repertoire. Nature 2012;486: 554-558.

8 Ostrov D, Grant B, Pompeu Y, et al: Drug hypersensitivity caused by alteration of the MHC-presented self-peptide repertoire. Proc Natl Acad Sci USA 2012;109:9959-9964.

9 European AIDS Clinical Society: European Guidelines for Treatment of HIV-Infected Adults in Europe. 2015, version 8.0.

10 Orkin C, Wang J, Bergin C, et al: An epidemiologic study to determine the prevalence of the HLA-B*5701 allele among HIV-positive patients in Europe. Pharmacogenet Genomics 2010;20:307-314

11 Mallal S, Nolan D, Witt C, et al: Association between presence of HLA-B*5701, HLA-DR7, and HLA-DQ3 and hypersensitivity to HIV-1 reverse transcriptase inhibitor abacavir. Lancet 2002;359:727-732.

12 Zerjav S, Fridman V, Suvakovic V, Jankovic T, Jevtovic D: Epidemiologija AIDS-a u Beogradu (in Serbian). Srp Arh Celok Lek 1987;115: 715-723.

13 European Centre for Disease Prevention and Control/WHO Regional Office for Europe: HIV/AIDS Surveillance in Europe 2014. Stockholm, European Centre for Disease Prevention and Control, 2015.
14 Stanojevic M, Siljic M, Salemovic D, et al: Ten years survey of primary HIV-1 resistance in Serbia: the occurrence of multiclass resistance. AIDS Res Hum Retroviruses 2014;30:634-641.

15 Jevtović D, Dragovic G, Salemović D, Ranin J, Kušić J, Marinković J: Treatment outcome of HAART-treated patients in a resource-limited setting: the Belgrade Cohort Study. Biomed Pharmacother 2014;68:391-395.

16 Hughes S, Hughes A, Brothers C, Spreen W, Thorborn D; CNA106030 Study Team: PREDICT-1 (CNA106030): the first powered, prospective trials of pharmacogenetic screening to reduce drug adverse events. Pharm Stat 2008; 7:121-129.

17 Saag M, Balu R, Phillips E, et al; Study of Hypersensitivity to Abacavir and Pharmacogenetic Evaluation Study Team: High sensitivity of human leukocyte antigen-B*5701 as a marker for immunologically confirmed abacavir hypersensitivity in white and black patients. Clin Infect Dis 2008;46:1111-1118.

18 Norcross M, Luo S, Lu L, Boyne M, et al: Abacavir induces loading of novel self-peptides into HLA-B*57:01: an autoimmune model for HLA-associated drug hypersensitivity. AIDS 2012;26:F21-F29.

19 Stocchi L, Cascella R, Zampatti S, Pirazzoli A, Novelli G, Giardina E: The pharmacogenomic HLA biomarker associated to adverse abacavir reactions: comparative analysis of different genotyping methods. Curr Genomics 2012;13: 314-320.

20 Orkin C, Sadiq ST, Rice L, Jackson F; UK EPI Team: Prospective epidemiological study of the prevalence of human leukocyte antigen (HLA)-B*5701 in HIV-1-infected UK subjects. HIV Med 2010;11:187-192.

21 Gonzalez-Galarza FF, Takeshita LY, Santos EJ, et al: Allele Frequency Net 2015 update: new features for HLA epitopes, KIR and disease and HLA adverse drug reaction associations. Nucleic Acids Res 2015;43:D784-D788.

22 Tumanov A, Laga V, Kazennova O, et al: The prevalence of the HLA-B*5701 allele in the European part of the Russian Federation. 12th European AIDS Conference/EACS. HIV Med 2009;10(suppl 2):84.

23 Grgic I, Zidovec-Lepej S, Planinic A, Gorenec L, Begovac J: The prevalence of HLA-B*5701 associated to hypersensitivity to abacavir in HIV-1-positive individuals from Croatia. Ther Pharmacol Clin Toxicol 2012;16S1:33-34.

24 Parczewski M, Leszczyszyn-Pynka M, Wnuk $\mathrm{A}$, et al: Introduction of pharmacogenetic screening for the human leucocyte antigen (HLA) B*5701 variant in Polish HIV-infected patients. HIV Med 2010;11:345-348.

25 Jilich D, Vrana M, Snopkova S, et al: Prevalence of human leukocyte antigen HLAB*57:01 in HIV-infected subjects in the Czech Republic. Cent Eur J Public Health 2011;19: 128-130.

26 Nikolic V, Salemovic D, Jevtovic D, et al: The prevalence of RT 245 codon polymorphisms and its association with duration of infection among HIV-1 patients in Serbia. BMC Infect Dis 2014;14(suppl 4):P4.

27 Andric Z, Popadic D, Jovanovic B, Jaglicic I, Bojic S, Simonovic R: HLA-A, -B, -C, -DRB1 and $-\mathrm{DQB} 1$ allele and haplotype frequencies in the Serbian population. Hum Immunol 2014; 75:218-226

28 Xiong $\mathrm{H}$, Huang J, Rong X, et al: HLA-B alleles $B^{*} 15: 01$ and $B^{*} 15: 02$ : opposite association with hepatitis $\mathrm{C}$ virus infection in Chinese voluntary blood donors. Intervirology 2015;58:80-87.

29 Kromer J, Hummel T, Pietrowski D, et al: Influence of HLA on human partnership and sexual satisfaction. Sci Rep 2016;6:32550.

30 Dello Russo C, Lisi L, Fabbiani M, Cauda R, Navarra P: Detection of HLA B*5701 by realtime PCR: implementation into routine clinical practice, additional validation data and comparison with commercially available methods. N Microbiol 2013;36(S1):193-194.

31 Vukmirovic D (ed): Women and Men in the Republic of Serbia. Belgrade, Statistical Office of the Republic of Serbia, 2014.

32 Hughes DA, Vilar FJ, Ward CC, Alfirevic A, Park BK, Pirohamed M: Cost-effectiveness analysis of HLA B*5701genotyping in preventing abacavir hypersensitivity. Pharmacogenetics 2004; 14:335-342.

33 Schackman BR, Scott CA, Walensky RP, Losina E, Freedberg KA, Sax PE: The cost-effectiveness of HLA-B*5701 genetic screening to guide initial antiretroviral therapy for HIV. AIDS 2008;22:2025-2033.

34 Kapoor R, Martinez-Vega R, Dong D, et al: Reducing hypersensitivity reactions with HLAB*5701 genotyping before abacavir prescription: clinically useful but is it cost-effective in Singapore? Pharmacogenet Genomics 2015; 25:60-72.

35 To SW, Chen JH, Wong KH, Chan KC, Tsang OT, Yam WC: HLA-B*5701 genetic screening among HIV-1 infected patients in Hong Kong: is this a practical approach in Han-Chinese? Int J STD AIDS 2013;24:50-52.
HLA-B*57:01 Allele in HIV-Infected Serbians
Intervirology 2017;60:43-47 DOI: $10.1159 / 000477810$ 